\title{
SOBRE LAS VIRTUDES Y LOS VICIOS DE LA ECONOMÍA
}

\author{
Raphael Bergoeing \\ CEP / Universidad de Chile
}

"La respuesta correcta en economía casi siempre es: depende".

"Es un modelo, no el modelo".

"Los modelos son una fuente de orgullo (para los economistas)... Para sus críticos (no economistas), los modelos capturan casi todo lo que está

mal con esta profesión".

Dani Rodrik, Economic Rules. ${ }^{1}$

\section{CONSTRUYENDO UN PUENTE}

D ani Rodrik es un economista influyente y provocador. Ha enseñado economía en la Escuela de Gobierno de la Universidad de Harvard durante casi tres décadas, ha publicado en las principales revistas científicas especializadas y es expositor habitual en los foros internacionales sobre política pública. Pero no es un economista tradicional: es parte de un reducido grupo de académicos que cuestionan el consen-

Raphael Bergoeing. PhD en economía, University of Minnesota. Investigador del Centro de Estudios Públicos y profesor asociado del Departamento de Ingeniería Industrial de la Universidad de Chile. Email: rbergoeing@cepchile.cl.

1 Dani Rodrik, Economics Rules: The Rights and Wrongs of the Dismal Science (Nueva York: Norton \& Company, 2015). Este libro será citado, de aquí en adelante, tan sólo con el número de página entre paréntesis. 
so pro liberalización, ${ }^{2}$ y apoya un tema tabú, la política industrial, que impulsa el desarrollo gubernamental de sectores específicos. En ambas materias, la opinión es mayoritariamente contraria. De hecho, pocas ideas son más ampliamente aceptadas entre los economistas que la necesidad de avanzar rápida y decididamente hacia un intercambio global. Sería la combinación de mercado y apertura comercial, profundizada desde la consolidación de la revolución industrial a mediados del siglo XIX, lo que definiría el enorme avance en prosperidad logrado por la humanidad durante los últimos 120 años. ${ }^{3}$ En cuanto a las políticas de desarrollo industrial, si bien recientemente ha habido un nuevo impulso, el peso de la prueba sigue en el Estado y, ante la duda, los economistas optan por no innovar. Pero Rodrik, desde una sala de clases o recorriendo el mundo asesorando a gobiernos de países en desarrollo, las apoya sin matices.

Por ello, cuando comencé a leer su nuevo libro, Economic Rules: The Rights and Wrongs of the Dismal Science, esperaba encontrarme con un texto cargado de críticas a esta disciplina científica, empático con el acuerdo popular reciente, especialmente después de la crisis financiera de 2008, que responsabiliza a la economía y a los economistas por no haber predicho la crisis, ni saber qué hacer para impedir que alguna similar volviese a suceder en el futuro.

Pero me equivoqué. El libro de Rodrik enjuicia a los economistas, especialmente a los académicos, por cómo hemos asumido nuestro rol en el ámbito de las políticas públicas. Sin embargo, sus juicios no son los de un intruso ni los de un opositor: los construye desde dentro de la disciplina, pidiendo cambios que evitarían los errores cometidos, pero, al mismo tiempo, defendiendo con convicción la metodología que la economía aplica y que tiende a alejarla de otras ciencias sociales.

${ }^{2}$ En su libro Has Globalization Gone too Far? (Washington D.C: Institute for International Economics, 1997), Rodrik plantea que la internacionalización de los mercados de bienes, servicios y capital abre una brecha entre, por un lado, los grupos cosmopolitas, profesionales y capacitados que pueden aprovecharla, y, por otro, el resto de la sociedad. Ello fomenta que emerjan tendencias populistas de derecha que defienden las políticas de identidad, como las impulsadas por Donald Trump en Estados Unidos; y de izquierda, que se enfocan en la mayor desigualdad, como las que promueve Bernie Sanders.

${ }^{3}$ Desde 1900, el producto per cápita mundial se ha multiplicado por seis, las expectativas de vida, por dos y las horas trabajadas han caído a la mitad. 
Así, este libro busca construir un puente entre economistas y no economistas, y está en consecuencia escrito para ambos públicos. El elemento con el que arma este puente son los modelos. Por un lado, los modelos representan el orgullo de los economistas. Son su manera de llevar la realidad al laboratorio, de analizar problemas concretos en un ambiente controlado, de incorporar rigor en el análisis, de proponer políticas con base científica. Para los críticos de la economía, por su parte, los modelos representan todo lo que está mal con esta disciplina: la obsesión con transformar el comportamiento humano, complejo y de libre albedrío, en proposiciones exactas, como físicos explicando el comportamiento de los átomos; el medio a través del cual se disfrazan ideologías como verdades científicas y se impone la teoría a la realidad. Una parte importante de este libro es, por lo tanto, sobre el necesario uso así como el costoso abuso de los modelos y la teoría en economía.

El momento para su publicación es, además, oportuno, cuando las virtudes de la economía han sido exageradas por muchos a la vez que otros tantos permanecen ciegos ante sus beneficios. Hoy, demasiadas personas relacionan los errores de economistas - y en ocasiones de expertos financieros como los que trabajan en Wall Street- con debilidades de la economía y, por extensión, del liberalismo económico.

Por una parte, no cabe duda de que hay mucho por mejorar en esta ciencia, especialmente dado que la economía ha tendido a funcionar en silos. Porque si bien el contenido de un buen programa de doctorado en economía incluye las diversas áreas que conforman la disciplina y se enseña de la misma manera en prácticamente todas las universidades, los académicos e investigadores han tendido a especializarse en extremo, concentrándose durante largos periodos de tiempo en mercados y preguntas puntuales. Por ejemplo, un economista interesado en temas monetarios típicamente no participa del debate ni conoce los avances en la discusión sobre competencia, y un economista laboral poco se interesa sobre los determinantes del mercado de capitales. Pero las crisis recientes, especialmente las financieras, han involucrado una compleja interacción entre mecanismos de mercado y regulatorios, tanto a nivel sectorial como agregado. Por ello, hoy existe consenso sobre la necesidad de que los macroeconomistas aprendan más de finanzas, de que los expertos en finanzas entiendan mejor cuándo los mercados dejan de 
funcionar, y todos tengan más conciencia sobre el rol de las burbujas de precios de activos y qué ocurre cuando éstas explotan.

Sin embargo, aunque los economistas estén en deuda, concluir que los políticos lo harán mejor al diseñar políticas públicas puede ser un corolario peligroso. El libro de Rodrik es un buen llamado a la mesura, a reconocer los límites de la ciencia, pero también las posibilidades que ofrece — a saber, qué esperar y qué no-; y a complementar la contribución de distintas disciplinas para avanzar hacia mejores políticas públicas.

El libro está escrito de manera amena, con buena pluma y múltiples ejemplos ilustrativos, que ayudarán al lector no experto. Sin embargo, en ocasiones también se torna repetitivo y abusa de la discusión metodológica. De hecho, cuatro de los seis capítulos están dedicados casi por completo a ello. Desde allí, Rodrik plantea su propuesta para mejorar la contribución de la economía a la política pública. El foco estaría en entender que los modelos en economía se acumulan horizontalmente - es decir, sumándose en lugar de reemplazándose-, agregando conocimiento que debe ser adecuadamente utilizado, esto es, mediante la elección del modelo correcto para cada pregunta, lo que, según reconoce el mismo autor, tiene más de arte que de ciencia $(21 ; 83)$. Así, los críticos habrían estado cuestionando a la economía por las razones equivocadas. Ningún modelo es correcto. Estos, por definición, son abstracciones de la realidad. En sus palabras, "los modelos nunca son verdaderos, pero hay verdad en los modelos" (44).

En este contexto, Rodrik aprovecha también este libro para difundir una metodología que ha desarrollado durante las últimas dos décadas para asesorar a gobiernos preocupados por el estancamiento económico. Esta herramienta, llamada "diagnóstico de crecimiento" ("growth diagnostics"), busca identificar las barreras principales para el crecimiento en un país y momento del tiempo específicos. La idea es que cada realidad necesita su propio diagnóstico, y él prioriza políticas al determinar qué debe ser solucionado primero. Esto lo ha apartado del Consenso de Washington, popularizado a fines de la década de 1980, cuando un conjunto ampliamente acordado de políticas liberalizadoras fue promovido desde los organismos multilaterales - Fondo Monetario Internacional y Banco Mundial, especialmente- e impuesto de manera general en América Latina. 
En otras palabras, si bien Rodrik es un defensor del método científico, es también un difusor de un mecanismo específico, diseñado para adaptarse a cada realidad con el fin de asumir desafíos particulares. Cuesta creer - apelando a un concepto económico básico- que los incentivos no estén mal alineados. Como el terapeuta que decide cuándo termina la terapia y el consultor que siempre termina su informe recomendando una consultoría adicional, Rodrik plantea que cada realidad es suficientemente única como para requerir un modelo único y un set de recomendaciones ad hoc, los que, además, no son trivialmente reconocibles, salvo desde la experiencia acumulada tras muchas jornadas en esas lides. Y eso, en una disciplina en la que el contexto importa, llevaría a descartar un modelo universal, algo que erradamente muchos habrían intentado imponer durante las últimas décadas. Así, hay mucha - demasiada - coherencia entre su crítica a la manera como muchos economistas han desarrollado su trabajo y su proyecto profesional de asesorías gubernamentales. De esta manera, la claridad con la que defiende una y otra vez a la disciplina económica, apoyando la importancia del método científico, incluso para entender el comportamiento humano, se nubla cuando debe justificar, desde el mismo rigor científico, la metodología que pretender impulsar.

A pesar de esto, Economics Rules es un libro muy recomendable y cuya lectura probablemente disfrutarán economistas y no economistas por igual.

En la sección siguiente se analizan, más detalladamente, los distintos capítulos de este libro. Luego, en la sección final, se contextualiza la discusión y se plantean algunas reflexiones sobre la enseñanza de la economía y la necesidad de combinar su campo de acción con otras disciplinas sociales, para mejorar el diseño e implementación de las políticas públicas.

\section{EL LIBRO Y SUS CAPÍTULOS}

El desencanto ciudadano con la economía, decíamos, ha crecido desde la crisis financiera subprime. Pero no sólo debido a su incapacidad de predecir. Los detractores de esta disciplina reclaman por la simpleza y falta de realidad de sus modelos, el supuesto sesgo pro mercado de sus propuestas y la tendencia a considerar el comportamiento huma- 
no motivado por objetivos parciales, ajenos a la cultura y dimensiones sociales. Dani Rodrik, sin embargo, es crítico de los economistas más que de la economía. En consecuencia, destina la mayor parte de su libro a los que enjuician esta disciplina, intentado explicarles por qué sus opiniones serían inadecuadas. Los primeros cuatro capítulos, de un total de seis, justifican, con ejemplos simples, el esfuerzo de la economía por avanzar desde el rigor del método científico. Recién en el capítulo 5 el libro asume una crítica frontal contra lo que considera es un sesgo en la profesión por privilegiar un tipo de modelos — pro mercado- sobre otros. Pero luego, en el capítulo 6 y final, vuelve a interpelar - aunque siempre con un lenguaje amigable - a los que se quejan contra la economía, explicando por qué, una a una, sus críticas estarían equivocadas.

En particular, en el primer capítulo, Rodrik explica para qué sirven los modelos y la relevancia de los supuestos simplificadores y de las matemáticas. Su argumento es como sigue: los modelos, al reducir la realidad, permiten entender fenómenos complejos en contextos específicos. Así, "cuando los modelos son elegidos juiciosamente, son una fuente de iluminación. Cuando son usados dogmáticamente, llevan a arrogancia y errores de política" (11). El objetivo es convencer al lector que más complejidad no es la respuesta. En este capítulo, además, aparece la tesis principal del libro, que consiste en que en economía se necesitan muchos modelos, con supuestos distintos, para ser utilizados en contextos particulares. De manera que "un buen economista" sería aquel que sabe cómo elegir el modelo más adecuado en cada caso (21).

Los capítulos 2 y 3 - "La ciencia del modelamiento económico" y "Navegando entre modelos", respectivamente - explican cómo la economía utiliza modelos, por qué ésta es una ciencia, si bien social, y cuál es su diferencia con ciencias naturales como la física y la química, pensadas desde leyes universales. Aclara, además, qué se puede esperar y qué no del análisis económico académico. Al ser una ciencia social, la economía no ofrece predicciones exactas, más bien acota las posibilidades del comportamiento humano, permitiendo aproximar el impacto de políticas públicas en distintos contextos sobre los consumidores, inversionistas y productores, entre otros. La economía, por lo tanto, no ofrecería teorías absolutas. En el mejor de los casos, se podría hablar de "tendencias, regularidades específicas al contexto bajo análisis y consecuencias probables" (45). 
Con todo, los modelos clarifican las hipótesis, imponen métodos empíricos y ayudan a producir nuevo conocimiento basado en estándares compartidos y acordados. Pero es en este mismo sentido que el planteamiento de Rodrik es ambiguo: si la economía no puede aspirar a modelar leyes universales, entonces no puede ser considerada ciencia. Porque si una cosa es no contar con un modelo único de aplicación total, otra es descartar la pretensión universal de la teoría. De hecho, la física clásica (o mecánica newtoniana) y la mecánica cuántica divergen en sus campos de aplicación, pero la aspiración científica es lograr construir un puente entre ellas. Al menos ésa es la aspiración científica. De la misma forma, y aunque todavía no tengamos modelos en economía para representar adecuadamente las crisis financieras, las teorías existentes han permitido avanzar significativamente en su comprensión. $\mathrm{Y}$ ese proceso de avance científico debería continuar durante los años venideros.

En estos capítulos, además, se explica cómo verificar cuáles son los supuestos críticos en un modelo, los mecanismos principales y sus implicancias incidentales. Esta tarea es compleja, y define la que parece ser una importante debilidad de la enseñanza de esta profesión: pese a lo determinante que serían el buen juicio y la experiencia para elegir el modelo adecuado a un contexto específico, la formación de economistas dedica poco o nada de tiempo y esfuerzo a desarrollar capacidades para ello.

Una parte sustancial del capítulo 3 lo dedica a explicar su método para elegir el modelo correcto, que consiste, tal como se mencionó, en el "diagnóstico de crecimiento".

Dado que para implementar una política pública adecuada sería necesario identificar cuál es el modelo apropiado al contexto específico, los principales errores ocurren cuando la ideología nubla el juicio o cuando los paradigmas se imponen a la realidad del contexto bajo análisis. Esta elección, por supuesto, tiene mucho de ciencia, pero también algo de arte, más si debe ser hecha en tiempo real. Con todo, lo mejor que podemos esperar es una aproximación tendencial y un impacto estimado.

En el capítulo 4, "Modelos y teorías", se recorre la evolución histórica de la economía y se explica cómo las teorías permiten estructurar el conocimiento ordenadamente, más que proveer respuestas concretas 
a preguntas específicas en contextos particulares. Sin embargo, esta revisión es excesivamente breve, por lo que no contribuye a entender lo mucho que ha cambiado esta disciplina y cómo esta evolución ha respondido a la acumulación natural de conocimiento, construyendo teoría desde la experiencia práctica. Rodrik desperdicia la oportunidad, por lo mismo, de contextualizar la relación virtuosa entre el avance de la teoría y el diseño de buenas políticas públicas. Un ejemplo exitoso es el de la teoría macroeconómica, que ha avanzado impactando directamente la manera como se organizan los bancos centrales, que hoy son autónomos del gobierno de turno en la mayoría de los países. Así también hay una relación directa entre la mayoría de las políticas públicas orientadas a controlar la inflación durante las últimas décadas y los desarrollos teóricos ocurridos durante los años 70 .

Parte importante de este capítulo consiste en explicar cómo identificar cuál modelo es más apropiado y entender las tendencias y consecuencias más probables de fenómenos y políticas específicas. El arte del uso de modelos se ejemplifica a través de múltiples ejemplos, y se insiste en la importancia de la experiencia acumulada y el buen juicio del responsable de esta tarea.

En los dos capítulos finales, más orientados a un público no especializado, se aterrizan las falencias de la disciplina y las principales críticas que ha recibido. En el capítulo 5, "Cuando los economistas se equivocan", se plantea que el error más común es confundir "un modelo con el modelo" (152). Para ejemplificarlo, como ya se dijo, se recurre al Consenso de Washington y, más recientemente, a la desidia regulatoria y excesiva confianza en el mercado que habrían llevado a la crisis financiera global de 2008. Así, se señala que el problema no es la economía, sino los economistas y su sesgo pro mercado. Una vez más, sin embargo, la alternativa a la aplicación de un conjunto de recomendaciones que han resultado ampliamente exitosas, como la liberalización comercial y el desarrollo productivo descentralizado (más que desde el Estado), no emerge como herramienta de política concreta, lo que genera un vacío entre crítica y propuesta.

Finalmente, en el capítulo 6, "La economía y sus críticos", se explica por qué el método científico es adecuado en economía, incluso cuando se trata de una disciplina social. Así, la simplificación de los modelos, sean estos construidos asumiendo mercados competitivos o 
no, no sería un inconveniente, siempre y cuando ellos se utilicen para responder preguntas acotadas en contextos en los que esos supuestos son útiles. Y dado que la economía trata sobre el comportamiento humano, no se puede esperar que los modelos entreguen predicciones precisas, sino más bien condicionadas, lo que permite una discusión sobre pilares técnicos objetivos y evaluables (184).

El principal problema del libro de Rodrik es su incoherencia al defender y criticar a la economía. Rodrik la defiende apelando al método científico, el que busca la construcción de ideas generales y de amplia aplicación, pero al mismo tiempo propone un método para asumir sus desafíos basado en casos absolutamente particulares. Y si todo es un caso particular, si no es posible establecer teorías de uso común, si cada problema requiere su propia solución y cada lugar y momento es una realidad en sí misma, se cae en la falacia que critica cuando defiende la simpleza de los modelos. El cuento de Borges que cita Rodrik, "Del rigor en la ciencia", sobre los mapas con escala 1:1, exactos pero inútiles, como parábola de la necesidad de aplicar el método científico es, a la vez, una crítica a la propuesta práctica del libro (43). ${ }^{4} \mathrm{Y}$ es que las diferencias entre países y entre momentos no impiden aplicar un conjunto amplio de ideas representadas a través de modelos cuyas implicancias han demostrado ser concretas.

La mayor preocupación, visto desde la política pública, es que la falacia de la indeterminación, como ocurre en muchas disciplinas sociales, transforme la necesidad de explorar en una justificación para no actuar. Hay personas, de carne y hueso, esperando beneficiarse de buenas políticas públicas. Hay desafíos sociales insoslayables y urgentes. La economía ha avanzado mucho y ha generado en la práctica herramientas para enfrentar estos desafíos. La incapacidad de replicar con perfección cada situación no es excusa para su mal uso, pero tampoco puede serlo para su no uso. La gran mayoría de los fracasos económicos recientes, especialmente en América Latina, son lisa y llanamente resultado del diseño e implementación de malas políticas públicas por parte de malos gobiernos, no de la incapacidad de los economistas de reconocer políticas específicas en contextos particulares.

${ }^{4}$ Jorge Luis Borges, "Del rigor en la ciencia", en El hacedor (Buenos Aires: Emecé, 1960). 
Con todo, el libro deja una sensación de dulce y agraz: dulce, por su claridad y compromiso con la difusión de un método y una disciplina muchas veces criticada desde la ignorancia, que ha contribuido enormemente a mejorar el bienestar de millones de personas durante los últimos dos siglos; y agraz, al criticar la ideologización de muchos economistas que, cumpliendo un rol como líderes de opinión, disfrazan sus preferencias como ciencia, pero sin ofrecer, desde el mismo rigor científico que demanda, una respuesta objetiva y evaluable sobre cómo éstos deberían asumir su tarea hacia adelante.

\section{REFLEXIONES FINALES}

Como el libro de Rodrik se inserta en un debate crítico de la economía, vale la pena intentar algunas reflexiones más sobre el tema.

Hay que comenzar por decir que el reparo actual a la economía no se limita a su rol durante la crisis financiera de 2008. El cuestionamiento es más profundo: se señala que el modelo económico de mercado, globalizado, imperante en Occidente durante gran parte del siglo pasado, habría fracasado. De hecho, hoy es habitual preguntarse cómo cambiarlo para asumir los desafíos que le esperan al mundo moderno. Estos reproches, si bien son más comunes entre los no economistas, han crecido también al interior de la profesión, con destacados académicos liderando el debate. ${ }^{5}$

Pero la mala reputación de la economía no es nueva. A mediados del siglo XIX, el historiador y filósofo escocés Thomas Carlyle la calificó como una "ciencia lúgubre" ("dismal science"), por su incapacidad para resolver el problema laboral que generaba la emancipación de los esclavos en las plantaciones de las Indias Occidentales. ${ }^{6}$ Más tarde, usó la misma expresión para referirse al futuro funesto que, según el reverendo Thomas Malthus, nos esperaba. ${ }^{7}$ Con recursos limitados, y ante la

${ }^{5}$ Un ejemplo es el Institute for New Economic Thinking, el que integra, entre otros, Joseph Stiglitz, profesor de la Universidad de Columbia y Premio Nobel de Economía 2001.

6 Thomas Carlyle, "Occasional Discourse on the Negro Question", Fraser's Magazine for Town and Country, vol. XL (1849): 672.

7 Thomas Carlyle, Chartism. Past and Present (London: Chapman and Hall, 1858). 
inexorable ley de rendimientos decrecientes, la expansión de la población no podría ser frenada sin producir miseria.

Defender a la economía exige enfrentar una complejidad mayor e importante: cuán rápido avanzan las ciencias, particularmente las sociales, y cómo se transforman en políticas concretas. En lo que sigue, se desarrolla esta idea y se reflexiona en torno a las principales críticas a esta disciplina y los desafíos que enfrenta para contribuir al diseño e implementación de mejores políticas públicas.

\section{La economía como ciencia}

La economía es una disciplina científica reciente. Por ejemplo, la macroeconomía, preocupada de las fluctuaciones agregadas de corto plazo, se origina a comienzos de la década de 1930, como reacción al alto y persistente desempleo que generó la Gran Depresión. La discusión previa se centraba en los temas que importaban para el financiamiento del Estado, principalmente el comercio internacional y la recaudación tributaria, y era asumida por los filósofos morales. Sólo a comienzos del siglo XIX aparecen los primeros cursos de economía enseñados por politólogos y economistas en las universidades europeas.

Que la economía sea una disciplina reciente significa que no debe extrañar su incapacidad para explicar fenómenos que, en lo cotidiano, son comunes. Y es que los tiempos de la ciencia y los de la vida práctica no son los mismos. Por ejemplo, en el ámbito de la astronomía, y pese a que el modelo de Copérnico, desarrollado a comienzos del siglo XVI, fue rápidamente aceptado como un instrumento teórico más elegante y apropiado que el modelo geocéntrico desarrollado por Tolomeo durante el siglo II, las técnicas de navegación continuaron basándose en la teoría de este último durante casi un siglo más.

La economía, como ciencia, trabaja con hechos y modelos. Los datos son obtenidos de la realidad habitual y, al ser sistematizados, revelan regularidades empíricas. Luego, con modelos se intenta replicar estas regularidades. Cuando un modelo parece apropiado, es utilizado para responder preguntas específicas. Por ejemplo, cuál es el efecto en el consumo privado de un aumento en la tasa de interés del Banco Central; o cuál es el impacto en la productividad agregada al liberalizar la economía; o qué pasa con los trabajadores poco calificados al aumentar el salario mínimo. 
Entonces, una cosa es la realidad, otra son los datos que intentan describirla y una tercera son los modelos que pretenden replicarla. Y en economía, por ser una ciencia social, surgen dos complicaciones particulares: trata sobre seres humanos, muchas veces impredecibles; y la mayor parte de las veces tiene relación con decisiones que dependen del futuro, casi siempre incierto. La economía debe, por lo tanto, predecir el comportamiento humano. Y esto, hasta ahora, no ha sido una tarea fácil.

Un ejemplo ilustra. El Banco Central es una institución, como pocas en Chile, que construye proyecciones con los más altos estándares técnicos. Comparemos sus predicciones para algunas variables clave con las cifras efectivamente observadas. Es trivial encontrar diferencias enormes entre ambas. Por ejemplo, a fines de 2014, el Informe de Política Monetaria (IPoM) - que el Banco Central publica trimestralmente - estimó para el año siguiente un crecimiento en torno al 3 por ciento y una inflación de 2,8. Los datos efectivos fueron 2,1 y 4,4 por ciento, respectivamente; esto es, sobrestimó el crecimiento en 30 por ciento y subestimó la inflación en 57 por ciento, ambos a doce meses. ${ }^{8}$

Por cierto, otras proyecciones han terminado siendo más atinadas. De hecho, cada vez que la variable proyectada no cambió significativamente entre un año y otro, el Banco Central acertó. La razón es obvia: cuando el comportamiento de las variables económicas se repite, las proyecciones aciertan; pero cuando las variaciones alcanzan magnitudes inusuales, aquéllas tienden a fracasar. $\mathrm{Y}$ es que las proyecciones económicas, alimentadas por la historia, nos hablan más del pasado que del futuro.

${ }^{8}$ Los errores en las proyecciones son comunes en todos los bancos centrales. De hecho, y como comparación con un país avanzado, Janet Yellen, presidenta de la Reserva Federal de Estados Unidos, señaló a mediados del año 2014 que esperaban subir su tasa de política monetaria hasta cuatro veces durante los doce meses siguientes. A comienzos de marzo 2015, sin embargo, en una audiencia ante el Congreso, dijo que no era descartable que la bajaran durante este año. La dificultad para acertar proyecciones macroeconómicas de corto plazo ha llevado a los bancos centrales a aumentar la frecuencia de sus intervenciones públicas, de modo de ir ajustando sus estimaciones ante los cambios que se generan en el entorno económico global. Durante la década de 1990, por ejemplo, era muy inusual que el presidente de un banco central hiciera declaraciones en la prensa. Hoy, sin embargo, estas instituciones tienen un calendario de entrevistas y presentaciones abiertas que aseguran intervenciones mensualmente. Al mismo tiempo, la información estadística que entregan al mercado ha crecido de manera significativa. 
Así, para que una proyección informe, ésta debe explicitar el grado de incertidumbre que la acompaña. Esta incertidumbre se representa a través de un intervalo de confianza. Por lo general, sin embargo, los medios de comunicación sólo publican el valor central de ese intervalo; algunas veces por simplicidad, otras porque el analista consultado no proporciona el rango.

Con el fin de ilustrar este concepto, se puede utilizar nuevamente la experiencia práctica del Banco Central de Chile. De acuerdo a sus IPoM, las proyecciones económicas son mucho menos precisas que lo que la discusión de coyuntura sugiere o hace aparecer. Según sus modelos, la desviación probable entre el centro del rango estimado y los valores observados es amplia. En el caso de la inflación, el Banco Central predice en su IPoM de marzo de 2015 que, con 90 por ciento de probabilidad, ésta se ubicará durante los próximos 18 meses entre 0 y 6 por ciento. Y en el caso del crecimiento, nuevamente con una confianza del 90 por ciento, la autoridad espera que la economía se expanda entre -1 y 7,5 por ciento durante el año 2017.

\section{Los economistas y Wall Street}

Hoy las opiniones de los economistas son recibidas con más escepticismo que antes, y eso está bien. Dado el nivel de incertidumbre ya mencionado, se debe sospechar de cualquier economista que plantee sus puntos de vista categóricamente.

Pero las críticas tienden a confundir el rol de los economistas académicos y el de los analistas profesionales, típicamente enfocados en el análisis coyuntural macroeconómico o en la predicción de precios de activos financieros. Estos últimos, muchas veces recuerdan al oráculo de Delfos que, más de 2.700 años atrás, se transformó en el primer servicio institucionalizado de proyecciones. Sus profecías eran astutamente reveladas: mediante un lenguaje ambiguo y confuso, se anunciaba al que consultaba lo que éste quería escuchar. De forma similar, en el mundo de las proyecciones económicas y financieras, éstas son generalmente fraseadas en forma condicional, con oscuro significado e imposibles de ser contrastadas con la realidad. Los cuánto, cuándo y cómo no abundan, y el análisis económico, que explica las causas y los efectos de los escenarios de riesgo enfrentados, tampoco. Así la cosa, poco contribuyen a la toma de decisiones. Con todo, el énfasis hacia proyeccio- 
nes libres de análisis sugiere que, en el terreno de los pronósticos, aún hay mucho por avanzar. El Banco Central entiende esto perfectamente; no es claro que la mayoría de los inversionistas privados que utilizan estas proyecciones también lo entiendan.

¿Significa esto que podemos liberarnos de las proyecciones? No mientras los agentes económicos deban planear sus estrategias en un entorno dinámico e incierto. Sí es necesario entender qué puede y qué no puede ser predicho, así como las ventajas y limitaciones de estas predicciones.

\section{La economía y el libre mercado}

Otra crítica reiterada es el supuesto sesgo pro mercado de la economía. Ella señala que las recomendaciones de política pública estarían desproporcionadamente inclinadas a soluciones descentralizadas, con un rol mínimo del Estado, y ello se explicaría por una presencia omnipresente del mercado en la enseñanza de la economía.

Pero la realidad es otra: un estudiante típico de economía accede a cursos enfocados en mercados no competitivos, con información incompleta, comportamiento estratégico, y un conjunto amplio de situaciones que justifican la intervención del Estado, en desmedro del rol del mercado. De hecho, el libro de texto más utilizado por estudiantes de economía en pregrado durante la segunda mitad del siglo XX, Economía: Un análisis introductorio, de Paul Samuelson, dedica más de un centenar de páginas al rol de Estado. La primera edición de este libro es de 1948. Desde entonces, además, la disciplina ha avanzado y los textos recientes incluyen numerosos tópicos adicionales a los considerados por Samuelson, con foco en las fallas de mercado. Esto se ha profundizado desde los años 80, cuando los modelos con competencia imperfecta y problemas de información fueron popularizados. Y más recientemente, lo mismo está ocurriendo con la preocupación por la desigualdad de ingresos.

Lo que ocurre, posiblemente, es que un problema común en los países pobres es la presencia de un Estado ineficiente y un mercado ahogado por regulaciones innecesarias. En el mundo desarrollado, sin embargo, esa dicotomía entre más Estado o más mercado carece de sentido y el foco está puesto en mejorar la calidad de ambos, lo que significa modernizar el Estado y fomentar la competencia dentro del mercado. 


\section{A modo de conclusión}

Hasta aquí se ha separado lo que hace la ciencia económica, como disciplina académica, y lo que el mundo de los negocios, y particularmente el mercado financiero, pretende. Se ha señalado, además, que la frontera del conocimiento es capaz de generar predicciones bastante menos certeras que lo que la prensa o incluso las presentaciones en seminarios públicos sugieren.

Una explicación posible para esta separación entre lo que la economía puede hacer y lo que los ciudadanos esperan de ella es la manera como muchos aprenden de economía. La mayoría de las personas acceden como máximo a uno o dos cursos básicos de economía en la universidad, y estos se enfocan en el modelo de competencia perfecta, una representación de la realidad en la que consumidores y productores se someten a las condiciones del mercado con información libre y abundantemente disponible, y en donde los poderes de unos y otros están desconcentradamente distribuidos. En este mundo, los precios y cantidades que resultan son, en un sentido preciso, socialmente óptimos. ${ }^{9}$ Esta es la base de una economía de mercado y sobre ella se construye el edificio de la disciplina.

En muchos mercados, la experiencia diaria de un consumidor y de un productor está bien representada por este modelo. Pero en la mayoría de los desafíos de política pública actuales, al enfrentar crisis financieras globales o ejemplos de colusión en mercados productivamente concentrados, los problemas son otros.

Por ello, los temas económicos que copan la discusión en los medios tratan sobre anomalías más que sobre los ejemplos del buen funcionamiento de la mayoría de los mercados. Lo que funciona bien pasa inadvertido, y lo que la mayoría de los no economistas estudió en cursos básicos no tiene relación con el debate en los medios. De hecho, hoy hay menos crisis que en el pasado, y cuando éstas ocurren son menos intensas; la inflación ha caído a niveles nunca antes vistos; y la liberación y el mercado globalizado han generado crecimiento sostenido, mejorando significativamente la prosperidad de miles de millones

${ }^{9}$ En economía, una producción y consumo son definidos como óptimos cuando cumplen el criterio de Pareto, esto es, cuando no es posible redistribuir estas cantidades mejorando a alguno sin empeorar a otro. 
de personas. La capacidad para generar un crecimiento alto y estable, que multiplicó el producto disponible seis veces durante el último siglo, mientras las horas trabajadas cayeron a la mitad de las observadas a comienzos de 1900, no había ocurrido antes en la historia de la humanidad. La preocupación original de Malthus por la hambruna resultó al revés. Hoy, según la Organización Mundial de la Salud, la principal causa de muerte global es la obesidad. Malthus predijo el fin de la humanidad por la falta de comida, pero es su exceso lo que nos está matando. ${ }^{10} \mathrm{La}$ pobreza material es, en la mayor parte de los países, resultado de problemas políticos e institucionales, reflejados en la falta de derechos de propiedad, la existencia de corrupción o inestabilidad política, pero no de escasez económica. Y es que el mercado globalizado está acabando con la escasez, tal cual la entendieron y vivieron cientos de generaciones desde el comienzo de la vida en sociedad siete mil años atrás. La pobreza en el mundo, que superaba el 95 por ciento de la población total a comienzos del siglo XIX, y que se había mantenido así por siglos, hoy es de 15 por ciento, según estima el Banco Mundial. Y con la superación de la pobreza, ha sido posible enfocarse en otros desafíos, hasta hace poco totalmente omitidos: la desigualdad, la sustentabilidad ecológica y la protección de la identidad cultural de los pueblos, entre otros. En gran medida, todo esto se debe al avance de la teoría económica y su reflejo en mejores políticas. Pero muchos de estos temas se discuten en profundidad sólo en cursos avanzados, a los que acceden exclusivamente los que luego serán economistas.

A pesar de lo anterior, estas mejoras no significan que se hayan superado las crisis económicas. Y que la pobreza se haya reducido a mínimos históricos no significa que estas crisis económicas, especialmente cuando son financieras, no causen enormes trastornos, en algunos casos con un alto costo material para varias generaciones.

La crisis de 2008 ha sido, como toda crisis, una oportunidad para aprender más sobre los desequilibrios financieros y la necesidad de seguir avanzando hacia regulaciones y políticas que reduzcan aún más su ocurrencia e impacto.

Este acervo de conocimiento, así como las nuevas preocupaciones que el crecimiento ha permitido asumir, representan también un desafío

10 Thomas Robert Malthus, An Essay on the Principle of Population (1798; reimpreso, Oxford: Oxford World's Classics, 1999), 39-45. 
para la enseñanza de la economía. Hoy, en todas las universidades se discute más sobre desigualdad, y se plantea el desafío de combinar la economía con otras ciencias sociales, tradicionalmente ausentes de su análisis. Junto a las matemáticas, la estadística y la computación, hoy aparecen la psicología, la sociología y la politología. También deberían incorporarse la historia y filosofía. De hecho, hasta mediados del siglo pasado los políticos eran asesorados por historiadores y filósofos. Parte importante de ese rol hoy lo asumen los economistas, y se han generado expectativas desmedidas con respeto a su capacidad parar ofrecer soluciones concretas. Pero la sociedad tiene también la responsabilidad de haber elegido este camino. El resultado ha sido concentrarse en el corto plazo y obviar muchas de las tendencias de largo plazo. En el futuro, todas estas disciplinas deberán contribuir a diseñar e implementar mejores políticas públicas. Ésa es la tarea pendiente. EP 